\title{
Lentiviral vector-mediated RNA interference targeted against prohibitin inhibits apoptosis of the retinoic acid-resistant acute promyelocytic leukemia cell line NB4-R1
}

\author{
YANFENG LIU, PENGCHENG HE, MEI ZHANG and DI WU \\ Department of Hematology, the First Affiliated Hospital, School of Medicine, Xi'an Jiaotong University, \\ Xi'an, Shaanxi 710061, P.R. China
}

Received March 16, 2012; Accepted September 24, 2012

DOI: $10.3892 / \mathrm{mmr} .2012 .1105$

\begin{abstract}
To investigate the possibility of prohibitin (PHB) inhibition by lentiviral vector-mediated RNA interference (RNAi) and its influence on cell apoptosis in the retinoic acid-resistant acute promyelocytic leukemia cell line NB4-R1, a lentiviral vector encoding a short hairpin RNA (shRNA) targeted against PHB (pGCSIL-GFP-PHB) was constructed and transfected into the packaging cells 293T, and the viral supernatant was collected to transfect NB4-R1 cells. Quantitative real-time fluorescent PCR and western blotting were used to detect the expression levels of PHB. Flow cytometry and detection of enzymatic activity of caspase-3 by western blotting were employed to examine cell apoptosis. Our results provide evidence that the lentiviral vector pGCSILGFP-PHB was constructed successfully, and the PHB mRNA and the protein expression inhibitory rates were 90.3 and $95.8 \%$, respectively. When compared to the control group, the activity of caspase- 3 decreased significantly, which showed a $57.3 \%$ downregulation, and the apoptosis rate was reduced by $44.6 \%(\mathrm{P}<0.05)$. In conclusion, downregulation of the PHB gene may inhibit apoptosis of NB4-R1 cells, and it is speculated that this was at least partly due to the downregulation of caspase-3, and PHB may be a novel target for gene therapy for retinoic acid-resistant acute promyelocytic leukemia.
\end{abstract}

\section{Introduction}

Acute promyelocytic leukemia (APL) is the M3 subtype of acute myeloid leukemia (AML) and is characterized by an

Correspondence to: Dr Pengcheng He and Dr Mei Zhang, Department of Hematology, the First Affiliated Hospital, School of Medicine, Xi'an Jiaotong University, 277 Yanta West Road, Xi'an, Shaanxi 710061, P.R. China

E-mail: hepc@163.com

E-mail: zhangmei@medmail.com.cn

Key words: prohibitin, NB4-R1, acute promyelocytic leukemia, RNA interference, lentiviral vector, apoptosis, caspase-3 accumulation of abnormal promyelocytes in the bone marrow and a severe bleeding tendency (1). Five decades ago, APL was the most fatal type of acute leukemia and was considered to be essentially untreatable (2). With the application of anthracyclines, retinoic acid (ATRA), arsenic trioxide (ATO) and other drugs, the APL complete remission rate has been significantly improved (3-5). However, between 20 and 30\% of newly diagnosed APL patients treated with regimens based on the use of ATRA or ATO will develop disease recurrence or drug resistance. With the rapid development of molecular biology, molecular-targeted therapy has become the hotspot of cancer prevention.

Prohibitin (PHB), a potential tumor suppressor protein, has attracted increasing attention due to its relationship with the occurrence and development of tumors. Research has shown that PHB may play a marked anti-tumor effect by regulating cell proliferation, differentiation, apoptosis, and hormone or receptor activity. However, the impact of $\mathrm{PHB}$ on the acute promyelocytic leukemia cell line and the exact mechanism involved remain controversial. RNA interference (RNAi) is a cellular pathway for post-transcriptional gene silencing. The ability to manipulate RNAi in the laboratory is being exploited to develop novel therapies for human disease (6). Unlike traditional plasmid vectors, lentiviral vector-mediated RNAi achieves the ultimate goal of long-term inhibition of target genes through combining the efficient infection and integration characteristics of a lentiviral vector with RNA inhibition of specific homologous gene expression. This provides a powerful research tool for endogenous gene function investigation (7). In this study, we have generated shRNAs that targeted against PHB in order to observe the effect on the retinoic acid-resistant acute promyelocytic leukemia cell line NB4-R1 and to try to elucidate the mechanism involved.

\section{Materials and methods}

Cell culture. The retinoic acid-resistant acute promyelocytic leukemia cell line NB4-R1 was a gift from the Shanghai Second Medical College. The cells were cultured in RPMI-1640 (Gibco-BRL, Carlsbad, CA, USA) supplemented with $10 \%$ heated-inactivated fetal bovine serum at $37^{\circ} \mathrm{C}$ in a humidified incubator containing $5 \% \mathrm{CO}_{2}$. Cell viability was 
evaluated by the trypan blue dye exclusion assay and only cell suspensions that presented more than $95 \%$ viability were used.

Design and cloning of lentiviral shRNA vectors. The target shRNAs against the human PHB gene (GeneBank accession number NM_002634) for RNAi were designed using an internet application system (Invitrogen, Carlsbad, CA, USA) as follows: 5'-GAGTTCACAGAAGCGGTGGAA3'. A shRNA which had no significant homology to any known human genes (5'-TTCTCCGAACGTGTCACGT-3') was used as a negative control (8). Oligos were heated at $95^{\circ} \mathrm{C}$ for $5 \mathrm{~min}$ and then annealed at $37^{\circ} \mathrm{C}$ for $1 \mathrm{~h}$. The annealed sequence was ligated into the AgeI and EcoRI sites of pGCSIL-GFP (containing human U6 promoter) to generate a pGCSIL-GFP-PHB vector (Shanghai Genechem Co., Ltd., China), which was then transformed into E. coli. Positive recombinant clones were selected by PCR (upstream primer: 5'-CCTATTTCCCATGATTCCTTCATA-3'; downstream primer: 5'-GTAATACGGTTATCCACGCG-3') and DNA sequencing. The new recombinant lentivirus vector was produced by co-transfecting $293 \mathrm{~T}$ cells with the lentivirus expression plasmid and packaging plasmids (pHelper 1.0 and pHelper 2.0) with Lipofectamine 2000 (Invitrogen). Infectious lentivirus vector was harvested at $48 \mathrm{~h}$ post-transfection and then concentrated. The infectious titer was determined using the hole by hole dilution method to determine the GFP-tagged positive rate in $293 \mathrm{~T}$ cells.

Lentiviral vector infection. Cells were divided into 3 groups: CON (uninfected NB4-R1 cells), RNAi-NC (infection with negative control lentiviral vector) and RNAi-PHB (infection with pGCSIL-GFP-PHB lentiviral vector). Cells were cultured at a density of $6 \times 10^{5} /$ well in 6-well plates and infected with specific/negative control lentiviral vectors and $8 \mu \mathrm{g} / \mathrm{ml}$ polybrene (Sigma, St. Louis, MO, USA), at the multiplicity of infection (MOI) 20, according to the pre-experimental results. After incubation for $48 \mathrm{~h}$, cells were observed under the fluorescence microscope. The knockdown efficiency of PHB was analyzed by real-time quantitative PCR and western blotting.

Measurement of $\mathrm{PHB}$ by real-time quantitative PCR. After infection with lentiviruses for 4 days, the total RNA from each group of cells was extracted in TRIzol (Invitrogen) and quantified by an ultraviolet spectrophotometer (UVP, Upland, CA, USA) at a wavelength of $260 \mathrm{~nm}$. The reverse transcription (RT) reaction was performed with a RevertAid First Strand cDNA Synthesis kit according to the manufacturer's instructions. A total of $20 \mu \mathrm{l}$ of a solution containing $10 \mu \mathrm{l}$ SYBR-Green Real-time PCR Master mix (Takara, Japan), $2 \mu 1$ cDNA, $7.2 \mu \mathrm{l}$ water and $0.2 \mu \mathrm{mol} / 1$ sense and antisense primers was used. PCR cycles were as follows: initial denaturation at $94^{\circ} \mathrm{C}$ for $5 \mathrm{~min}$, the reaction was repeated for 40 cycles, each cycle consisted of denaturing at $94^{\circ} \mathrm{C}$ for $30 \mathrm{sec}$, annealing at $61^{\circ} \mathrm{C}$ for $45 \mathrm{sec}$, and synthesis at $72^{\circ} \mathrm{C}$ for $45 \mathrm{sec}$. The primers for amplifying $\mathrm{PHB}$ and $\mathrm{GAPDH}$ were as follows: GAPDH forward, 5'-CACCCTGTTGCTGTAGCCAAA-3', and reverse, 5'-CACCCTGTTGCTGTAGCCAAA-3'; PHB forward, 5'-CTGCCGTCCATCACAACTG-3' and reverse, 5'-TCTGTAAGGTCGTCGCTCAC-3'. The cutoff point $(\mathrm{Ct})$ of each sample was plotted on a standard curve and the mRNA

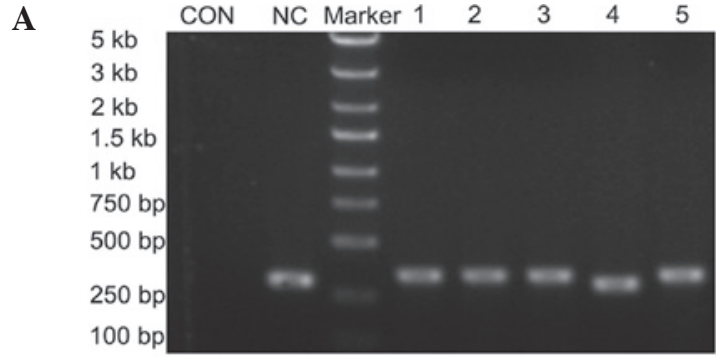

B
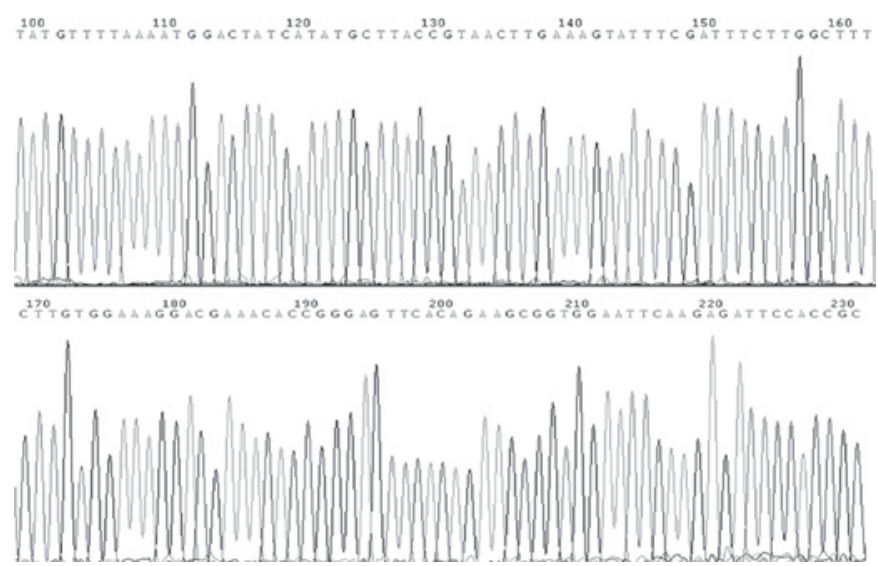

Figure 1. (A) Identification of recombinant lentiviral vectors by PCR. Lane 1, uninfected NB4-R1 cells; lane 2, recombinant negative control lentiviral vector; lane 3, marker ladder; lanes $1-5$, recombinant positive lentiviral vector with prohibitin (PHB) short hairpin RNA (shRNA). (B) Identification of recombinant lentiviral vectors by DNA sequencing.

copy numbers were calculated. The GAPDH gene was used as an endogenous control. The relative PHB mRNA levels were expressed as a ratio of PHB to GAPDH.

Measurement of PHB by western blotting. After infection with lentiviruses for 7 days, the cells were lysed in RIPA buffer in the presence of a proteinase inhibitor cocktail (Sigma). Protein concentration was determined using a BCA assay kit (Thermo, Waltham, MA, USA). Protein (30 $\mu \mathrm{g})$ was separated using $10 \%$ SDS-PAGE and transferred to nitrocellulose membranes. Membranes were blocked in 5\% skimmed milk for $2 \mathrm{~h}$, incubated with primary antibodies against PHB (mouse monoclonal, 1:700, Abcam, Cambridge, MA, USA) and GAPDH (mouse monoclonal, 1:10000, Santa Cruz Biotechnology, Inc., Santa Cruz, CA, USA) at room temperature for $2 \mathrm{~h}$ and $4^{\circ} \mathrm{C}$ overnight, washed with TBS containing $0.1 \%$ Tween-20 (TBST) followed by an incubation of $1 \mathrm{~h}$ in mouse anti-mouse secondary antibody conjugated with HRP. After the final wash with TBST, the membranes were developed using chemiluminescence and exposed to X-ray films. The immunoblots were quantified with Quantity One version 4.6.2 software. The expression of PHB in each sample was internally normalized to GAPDH and levels were given relative to the expression in control groups.

\section{Measurement of apoptosis}

Flow cytometric analysis. To measure the numbers and the ratio of apoptotic cells, the Annexin V-FITC Apoptosis Detection kit (BD Biosciences, Franklin Lakes, NJ, USA) was utilized. The cells were seeded at $1 \times 10^{5} /$ well in 24-well plates, 

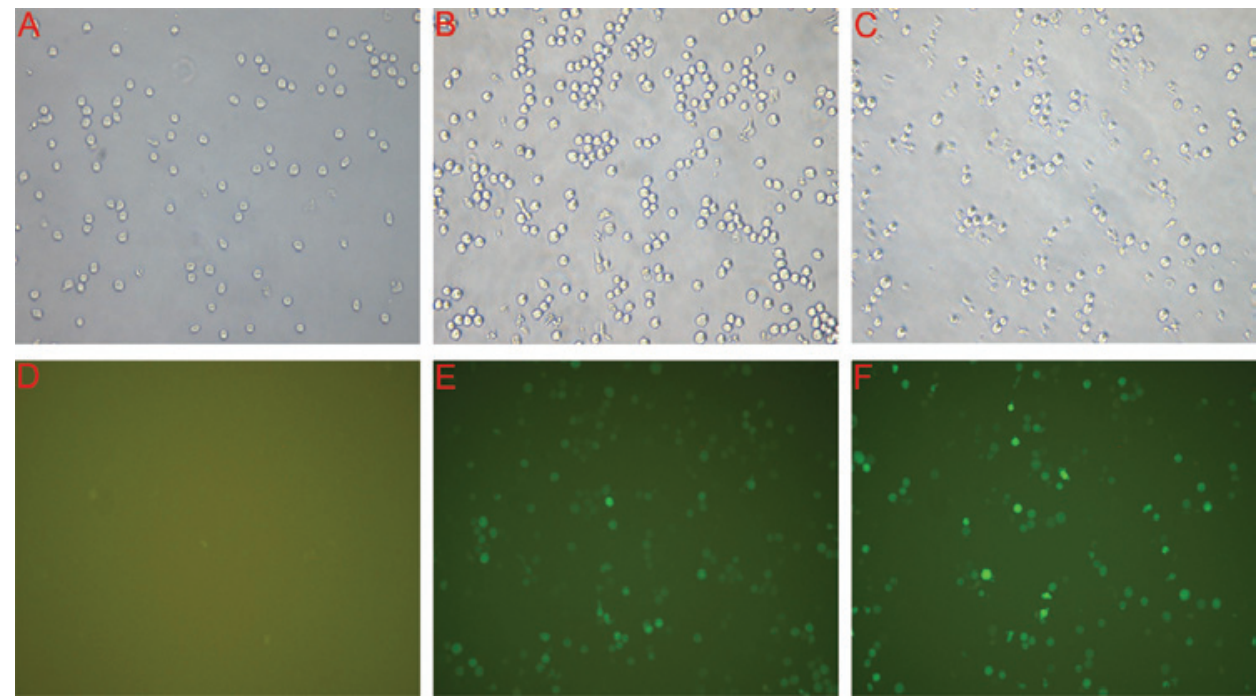

Figure 2. Fluorescence images of NB4-R1 cells following lentiviral vector infection (x100). (A and D) CON (uninfected NB4-R1 cells). (B and E) RNA interference (RNAi)-NC (NB4-R1 + negative control lentiviral vector). (C and F) RNAi-prohibitin (PHB) (NB4-R1 + lentiviral vector with short hairpin RNA of PHB).

and on the next day, they were transfected with RNAi-PHB or RNAi-NC, respectively. Seven days after transfection, the cells were harvested, washed twice with cold PBS, and resuspended in Annexin $\mathrm{V}$ binding buffer. The cell suspension was then transferred to a 5-ml centrifuge tube, and stained with $5 \mu 1$ Annexin V-Fitc at room temperature in the dark for $15 \mathrm{~min}$. Finally, the stained cells were run immediately on a FACSort Flow Cytometer (Becton-Dickinson) and evaluated with the CELLQuest software system (BD Biosciences).

Western blot analysis of caspase-3 protein level. The western blot analysis of caspase-3 levels was performed according to the caspase-3 (Mouse monoclonal, 1:1000; Cell Signaling Technology, Inc., USA) antibody description.

Statistical analysis. All the experiments were carried out in triplicate, and quantitative data in this study were expressed as $\bar{\chi} \pm$ s. Statistical differences between the groups were compared using a Student's t-test. $\mathrm{P}<0.05$ was considered to indicate a statistically significant difference.

\section{Results}

Construction and package of the lentiviral vector. The target shRNAs against human PHB for RNAi were constructed and connected with lentiviral frame plasmids. Positive recombinant clones were selected and identified with PCR. The sizes of PCR amplified fragments of recombinant clones and unconnected shRNA empty vectors were 343 and $306 \mathrm{bp}$, respectively (Fig. 1A). DNA sequencing analysis confirmed that the inserted PHB shRNA sequences were correct (Fig. 1B). The recombinant lentiviral vectors were applied to co-transfect 293T cells and virus titers were determined as $1 \times 10^{8}$ (pGCSIL-GFP-PHB lentiviral vector) and $5 \times 10^{8}$ (negative control lentiviral vector), respectively.

Infection efficiency of human NB4-R1 cells with lentivirus. Assessment of the infection rate was accomplished with the determination of the positive expression rate of GFP under
A

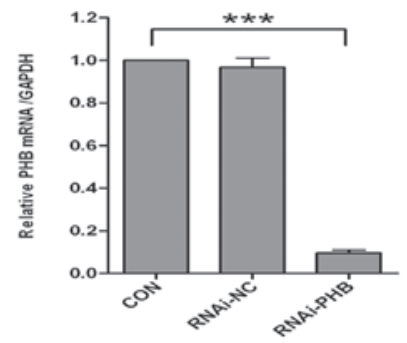

\section{B}
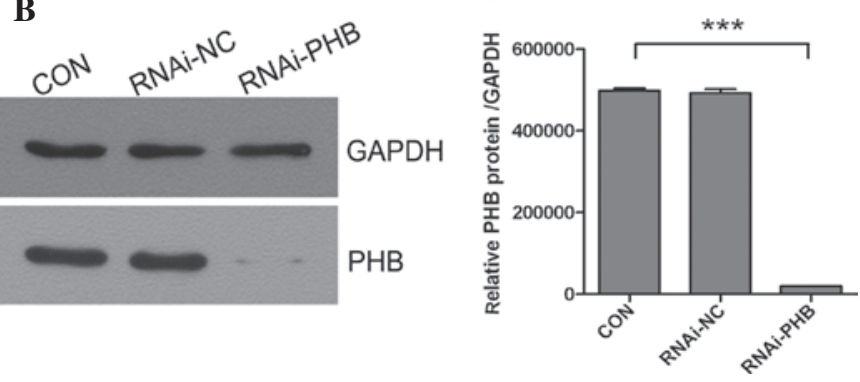

Figure 3. (A) Expression of prohibitin (PHB) mRNA in NB4-R1 cells after infection with lentivirus by real-time quantitative PCR. (B) Expression of PHB protein in NB4-R1 cells after infection with lentivirus by western blotting.
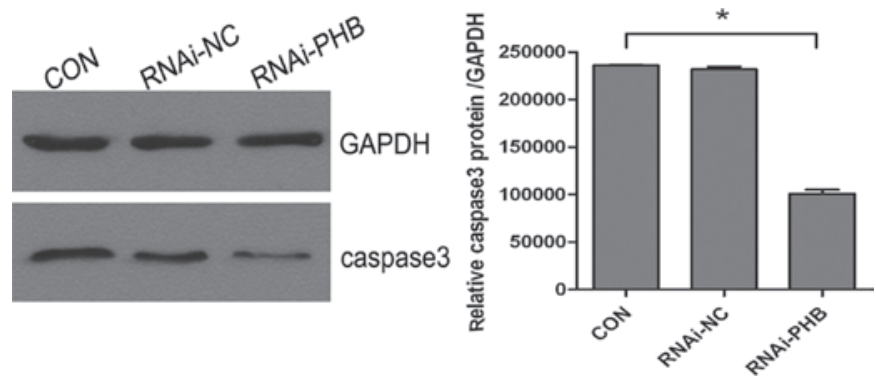

Figure 4. Detection of caspase-3 protein expression level by western blotting.

the fluorescent microscope 4 days after infection. As shown in Fig. 2, the infection efficiency of lentiviral RNAi vectors of PHB and negative controls in NB-R1 cells was $>80 \%$. 

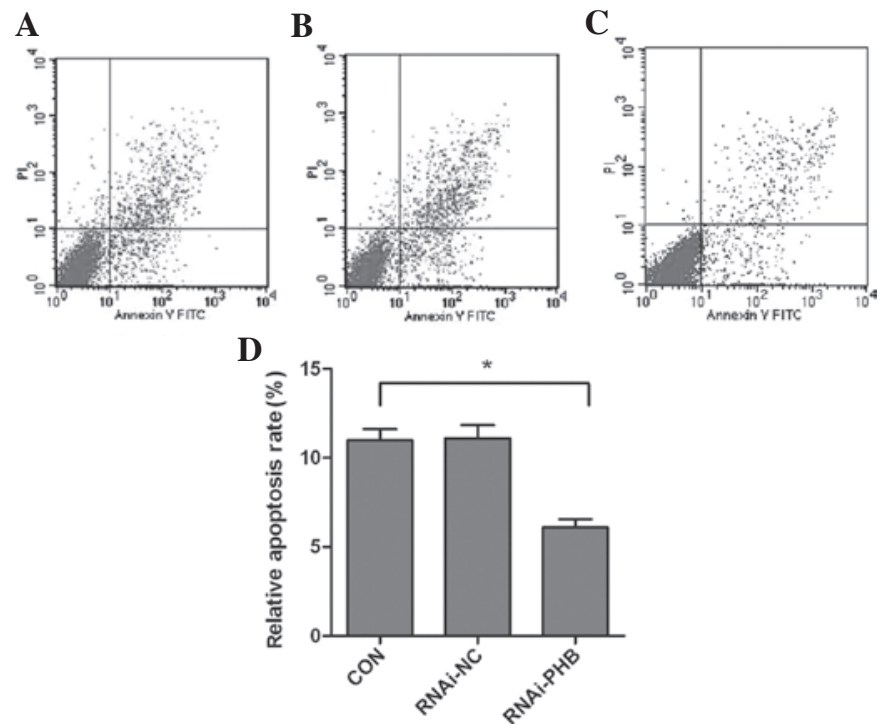

Figure 5. Effect of prohibitin (PHB) silencing on apoptosis of NB4-R1 cells. The number of apoptotic cells was determined seven days after infection with specific or negative control lentiviral vectors using flow cytometry. (A) Uninfected NB4-R1 cells. (B) NB4-R1 + negative control lentivira vector. (C) NB4-R1 + lentiviral vector with short hairpin RNA (shRNA) of PHB. (D) Summary of the experiment illustrated in (A-C). Error bars indicate $\bar{\chi} \pm \mathrm{s}$ of three replicates.

Silencing efficacy of PHB shRNA lentivirus. The silencing efficacy of PHB shRNA lentivirus at mRNA and protein levels in NB4-R1 cells was determined by real-time quantitative PCR and western blotting in CON, RNAi-NC and RNAi-PHB groups, respectively. The results showed that the expression levels of PHB in RNAi-PHB were markedly reduced by $90.3 \%$ (mRNA, Fig. 3A) and 95.8\% (protein, Fig. 3B) compared with those in the control group. Both real-time quantitative PCR and western blotting demonstrated that lentiviral vectors were effective for PHB silencing.

PHB gene silencing inhibits cell apoptosis. The effect of PHB silencing on the apoptosis of NB4-R1 cells was determined by FCM and western blot analysis of caspase-3. As shown in Fig. 4, when compared to the control group, the activity of caspase-3 decreased significantly, which showed a $57.3 \%$ downregulation, and the rate of apoptosis was reduced by approximately $44.6 \%$ (Fig. 5 ; $\mathrm{P}<0.05$ ).

\section{Discussion}

PHBs are ubiquitous, evolutionarily conserved proteins, which comprise two highly homologous subunits, termed PHB1 and PHB2. These two proteins assemble into a ring-like macromolecular structure and are implicated in diverse cellular processes, from mitochondrial biogenesis and function to cell cycle, signaling, proliferation, apoptosis and transcriptional control (9-11). In humans, the PHB gene has been located at q21 of chromosome 17 (10), and has been associated with various types of disease states, such as inflammation $(12,13)$, obesity and diabetes mellitus (14), Parkinson disease (15) and cancer $(16,17)$. Among them, PHB anti-tumor mechanisms are the focus of attention. At present, many studies have confirmed that human breast cancer (18), cervical and ovarian cancer (19), bladder cancer (20), rectum cancer, leukemia (21) and other solid tumor or non-solid tumor tissue cells have high expression of PHB. The biochemical function and specific mechanism, however, have remained largely elusive. Earlier reports showed that $\mathrm{PHB}$, as a potential tumor suppressor with various biological activity, was attributed to the 3'-UTR of mRNA. McClung et al found that wild-type 3'-UTR mRNA was capable of inhibiting the proliferation of tumor cells, while the mutant cells lose this activity (22). Other investigations have demonstrated the anti-tumor activities and evolutionary conservation of PHB, which has attracted the attention of researchers, resulting in the discovery of the association between PHB and the E2F pathway $(23,24)$. E2F activity is essential for the expression of critical cellular genes. By combining with E2F, PHB may collect a variety of transcription inhibitors and then suppress the transcriptional activity of the tumor, so that the cell cycle is arrested in the G1/S phase, achieving the purpose of inhibiting cell proliferation and inducing cell apoptosis $(25,26)$. Additional diverse functions of PHB were recently reported, which link this evolutionary highly-conserved gene to the induction of cancer cell apoptosis through the Raf-MEK-ERK cascade $(27)$, p53 $(28,29)$ and estrogen receptor (30). There is significant evidence to indicate that PHB may be a promising tumor-specific marker and drug therapy target.

RNAi is one of the most significant discoveries in the field of gene regulation and has broad development prospects in the research of tumor pathogeny, immune mechanisms and therapy $(31,32)$. Delivering siRNA into cells is the greatest impediment to RNAi therapy. There are two main mechanisms to achieve RNAi: delivering chemically synthesized siRNA into cells, which directly or continuously produce siRNAs by transcription in cells. Chemically synthesized siRNA could be introduced into cells via traditional delivery strategies; however, it has short gene silencing effects and cannot be passed to cell progeny. Therefore, in recent years, the attention of researchers has turned to expression vectors, which include retroviral, lentivirus and others, in order to synthesize siRNA in mammalian cells. In the past few years, shRNA has been widely used to silence the expression of many target genes due to its high specificity and apparent nontoxicity (33).

Since the lentivirus vectors have high efficiency for gene delivery, low immune reactions in vivo, and may be integrated into the non-dividing cell genome to achieve stable long-term expression (34), we constructed a lentiviral shRNA vector of the PHB gene. In this study, we found an effective lentiviral vector-mediated RNAi had been performed successfully. Moreover, accompanied by the downregulation of PHB, reduced expression of caspase-3 and lower apoptosis rates were observed. Caspases are crucial mediators of programmed cell death (apoptosis). Among them, caspase-3 is a frequently activated death protease, catalyzing the specific cleavage of many key cellular proteins. Caspase-3 is essential for major apoptotic scenarios in a remarkable tissue-, cell type- or death stimulus-specific manner. It is also indispensable for apoptotic chromatin condensation and DNA fragmentation in all cell types examined (35). Although the defined relationship and mechanism between PHB and NB4-R1 cell apoptosis remain largely unknown, 
our research may provide substantial information on the function of the PHB gene in APL and provide a new target of leukemia therapy.

\section{Acknowledgements}

This study was supported by the Natural Science Foundation of China (no. 30701133), the Fundamental Research Funds for the Central Universities and the Shaanxi Province Science and Technology Development Fund, China (2010K01-135, 2012KTCL03-12). The authors express their gratitude to Dr Xinyang Wang and Dr Wen Wen for their technological assistance.

\section{References}

1. Hu J, Liu YF, Wu CF, et al: Long-term efficacy and safety of all-trans retinoic acid/arsenic trioxide-based therapy in newly diagnosed acute promyelocytic leukemia. Proc Natl Acad Sci USA 106: 3342-3347, 2009.

2. Hillestad LK: Acute promyelocytic leukemia. Acta Med Scand 159: 189-194, 1957.

3. Bernard J, Weil M, Boiron M, Jacquillat C, Flandrin G and Gemon MF: Acute promyelocytic leukemia: results of treatment by daunorubicin. Blood 41: 489-496, 1973.

4. Wang ZY and Chen Z: Acute promyelocytic leukemia: from highly fatal to highly curable. Blood 111: 2505-2515, 2008.

5. Sanz MA: Arsenic trioxide in the management of APL: proceed with caution. Oncology (Williston Park) 25: 743, 746, 2011.

6. Gonzalez-Alegre P, Bode N, Davidson BL and Paulson HL: Silencing primary dystonia: lentiviral-mediated RNA interference therapy for DYT1 dystonia. J Neurosci 25: 10502-10509, 2005.

7. Ye X, Liu T, Gong Y, Zheng B, Meng W and Leng Y: Lentivirusmediated RNA interference reversing the drug-resistance in MDR1 single-factor resistant cell line K562/MDR1. Leuk Res 33: 1114-1119, 2009.

8. Rodriguez M, Aladowicz E, Lanfrancone L and Goding CR: Tbx3 represses E-cadherin expression and enhances melanoma invasiveness. Cancer Res 68: 7872-7881, 2008.

9. Artal-Sanz M and Tavernarakis N: Prohibitin couples diapause signalling to mitochondrial metabolism during ageing in C. elegans. Nature 461: 793-797, 2009.

10. Merkwirth $C$ and Langer T: Prohibitin function within mitochondria: essential roles for cell proliferation and cristae morphogenesis. Biochim Biophys Acta 1793: 27-32, 2009.

11. Týc J, Faktorová D, Kriegová E, Jirků M, Vávrová Z, Maslov DA and Lukes J: Probing for primary functions of prohibitin in Trypanosoma brucei. Int J Parasitol 40: 73-83, 2010.

12. Sharma A and Qadri A: Vi polysaccharide of Salmonella typhi targets the prohibitin family of molecules in intestinal epithelial cells and suppresses early inflammatory responses. Proc Natl Acad Sci USA 101: 17492-17497, 2004

13. Theiss AL, Idell RD, Srinivasan S, Klapproth JM, Jones DP, Merlin D and Sitaraman SV: Prohibitin protects against oxidative stress in intestinal epithelial cells. FASEB J 21: 197-206, 2007.

14. Kolonin MG, Saha PK, Chan L, Pasqualini R and Arap W: Reversal of obesity by targeted ablation of adipose tissue. Nat Med 10: 625-632, 2004.

15. Ferrer I, Perez E, Dalfó E and Barrachina M: Abnormal levels of prohibitin and ATP synthase in the substantia nigra and frontal cortex in Parkinson's disease. Neurosci Lett 415: 205-209, 2007.

16. Mengwasser J, Piau A, Schlag P and Sleeman JP: Differential immunization identifies PHB1/PHB2 as blood-borne tumor antigens. Oncogene 23: 7430-7435, 2004.
17. Gamble SC, Chotai D, Odontiadis M, Dart DA, Brooke GN, Powell SM, Reebye V, Varela-Carver A, Kawano Y, Waxman J and Bevan CL: Prohibitin, a protein downregulated by androgens, represses androgen receptor activity. Oncogene 26: 1757-1768, 2007.

18. Peng X, Mehta R, Wang S, Chellappan S and Mehta RG: Prohibitin is a novel target gene of vitamin $\mathrm{D}$ involved in its antiproliferative action in breast cancer cells. Cancer Res 66: 7361-7369, 2006

19. Gregory-Bass RC, Olatinwo M, Xu W, Matthews R, Stiles JK, Thomas K, Liu D, Tsang B and Thompson WE: Prohibitin silencing reverses stabilization of mitochondrial integrity and chemoresistance in ovarian cancer cells by increasing their sensitivity to apoptosis. Int J Cancer 122: 1923-1930, 2008.

20. Wu TF, Wu H, Wang YW, Chang TY, Chan SH, Lin YP, Liu HS and Chow NH: Prohibitin in the pathogenesis of transitional cell bladder cancer. Anticancer Res 27: 895-900, 2007.

21. Qi J, He P, Chen W, Wang H, Wang X and Zhang M: Comparative proteome study of apoptosis induced by As4S4 in retinoid acid resistant human acute promyelocytic leukemia NB4-R1 cells. Leuk Res 34: 1506-1516, 2010.

22. McClung JK, Jupe ER, Liu XT and Dell'Orco RT: Prohibitin: potential role in senescence, development, and tumor suppression. Exp Gerontol 30: 99-124, 1995.

23. Wang S, Nath N, Adlam M and Chellappan S: Prohibitin, a potential tumor suppressor, interacts with RB and regulates E2F function. Oncogene 18: 3501-3510, 1999.

24. Rastogi S, Joshi B, Dasgupta P, Morris M, Wright K and Chellappan S: Prohibitin facilitates cellular senescence by recruiting specific corepressors to inhibit E2F target genes. Mol Cell Biol 26: 4161-4171, 2006.

25. Wang S and Faller DV: Roles of prohibitin in growth control and tumor suppression in human cancers. Transl Oncogenomics 3: 23-37, 2008.

26. Singh S, Johnson J and Chellappan S: Small molecule regulators of $\mathrm{Rb}-\mathrm{E} 2 \mathrm{~F}$ pathway as modulators of transcription. Biochim Biophys Acta 1799: 788-794, 2010.

27. Ande SR, Xu Z, Gu Y and Mishra S: Prohibitin has an important role in adipocyte differentiation. Int J Obes (Lond) 36: 1236-1244, 2012.

28. Joshi B, Rastogi S, Morris M, Carastro LM, DeCook C, Seto E and Chellappan SP: Differential regulation of human YY1 and caspase 7 promoters by prohibitin through $\mathrm{E} 2 \mathrm{~F} 1$ and $\mathrm{p} 53$ binding sites. Biochem J 401: 155-166, 2007.

29. Fusaro G, Dasgupta P, Rastogi S, Joshi B and Chellappan S: Prohibitin induces the transcriptional activity of p53 and is exported from the nucleus upon apoptotic signaling. J Biol Chem 278: 47853-47861, 2003.

30. He B, Feng Q, Mukherjee A, Lonard DM, DeMayo FJ, Katzenellenbogen BS, Lydon JP and O'Malley BW: A repressive role for prohibitin in estrogen signaling. Mol Endocrinol 22: 344-360, 2008

31. Davis ME, Zuckerman JE, Choi CH, Seligson D, Tolcher A, Alabi CA, Yen Y, Heidel JD and Ribas A: Evidence of RNAi in humans from systemically administered siRNA via targeted nanoparticles. Nature 464: 1067-1070, 2010.

32. Moffat J, Grueneberg DA, Yang X, et al: A lentiviral RNAi library for human and mouse genes applied to an arrayed viral high-content screen. Cell 124: 1283-1298, 2006.

33. Gartel AL and Kandel ES: RNA interference in cancer. Biomol Eng 23: 17-34, 2006.

34. Cockrell A and Kafri T: Gene delivery by lentivirus vectors. Mol Biotechnol 36: 184-204, 2007.

35. Porter AG and Jänicke RU: Emerging roles of caspase-3 in apoptosis. Cell Death Differ 6: 99-104, 1999. 